\title{
Intra- and Interpersonal Movement Coordination in Jointly Moving a Rocking Board
}

\author{
Jurjen Bosga, Ruud G. J. Meulenbroek, Raymond H. Cuijpers
}

In this study, we investigate how two persons (dyads) coordinate their movements when performing cyclical motion patterns on a rocking board. In keeping with the Leading Joint Hypothesis (Dounskaia, 2005), the movement dynamics of the collaborating participants were expected to display features of a prime mover with low movement variability. Fourteen subject pairs performed the task in nine amplitude-frequency combinations that were presented in the form of a to-be-tracked stimulus on a computer display. Participants were asked to track the stimulus by jointly rocking the Board sideways while receiving continuous visual feedback of its rotations. Displacements of 28 IREDS that were attached to the rocking board, both ankles, knees, hips, shoulders and heads of both actors, were sampled at $75 \mathrm{~Hz}$ by means of a 3D-motion tracking system. From these data, we derived body-segment angular excursions as well as the continuous relative phase and time-lagged cross-correlations between relevant joint excursions. The results show that, at the intrapersonal level, knee rotations initially led all other joints in time while the antiphase coordination between the knees displayed relative low variability. At the interpersonal level, dyads adopted a leader-follower strategy with respect to the coordination demands of the task. We take that knee rotations create a dynamic foundation at both intra- and interpersonal levels involving subordination of individual action to joint performance thereby allowing for lowdimensional control of joint action in a high-dimensional, repetitive motor task.

Keywords: coordination, redundancy, leading joint, joint action

Research into between-subject (interpersonal) movement coordination is gradually shifting beyond the exploration of the tacit entrainment of rhythmic motion patterns in tasks that lack any shared action goal. For example, Knoblich and Jordan (2003) have shown in a tracking task that groups possess the ability to use and learn an anticipatory coordination strategy as long as they have access to visual feedback of their coactor's performance. Recently, we demonstrated in an isometric virtual-lifting task with real-time visual feedback that dyads were capable of resolving redundancy by administering force-sharing synergies (Bosga

Bosga and Meulenbroek are with the Donders Institute for Brain, Cognition and Behaviour Centre for Cognition, Radboud University, Nijmegen, The Netherlands. Cuijpers is with Human Technology Interaction, Eindhoven University of Technology, Eindhoven, The Netherlands. 
\& Meulenbroek, 2007). Even though group performance in these studies was slower than individual performance, the group always performed the task successfully. On the other hand, haptically linked dyads performed significantly faster than individuals on a target-acquisition task (Reed, Peshkin, Hartmann, Grabowecky, Patton \& Vishton, 2006). Although dyad members exerted large task-irrelevant counteracting forces, task completion times were systematically lower in dyads (see also Wegner \& Zeaman, 1956).

It is well established that in many motor tasks reafferent feedback forms a precondition for successful task performance (Carlton, 1981; Paillard, 1996). Therefore, a possible explanation for less proficient performance by dyads, as compared with individual performance in the aforementioned studies, is that dyads acted on relative slow on-line visual feedback ( 135 ms; Carlton, 1981; Saunders \& Knill, 2003) of their partners' actions to predict the consequences of their shared actions. Better performance of the haptically linked dyads in the latter study could indicate that they relied on relative fast haptic feedback ( $\sim 65 \mathrm{~ms}$; Johansson \& Birznieks, 2004) to coordinate their actions together.

We reasoned that if the consequences of the coactor's performance can indeed be picked up haptically, control principles that have been postulated for individual motor control might also be exploited in joint action. To evaluate this line of reasoning, we investigated how two mechanically linked persons mutually coordinate their movements while performing a repetitive motor task on a rocking board (see Figure 1). In general, the rocking board is used in physical rehabilitation

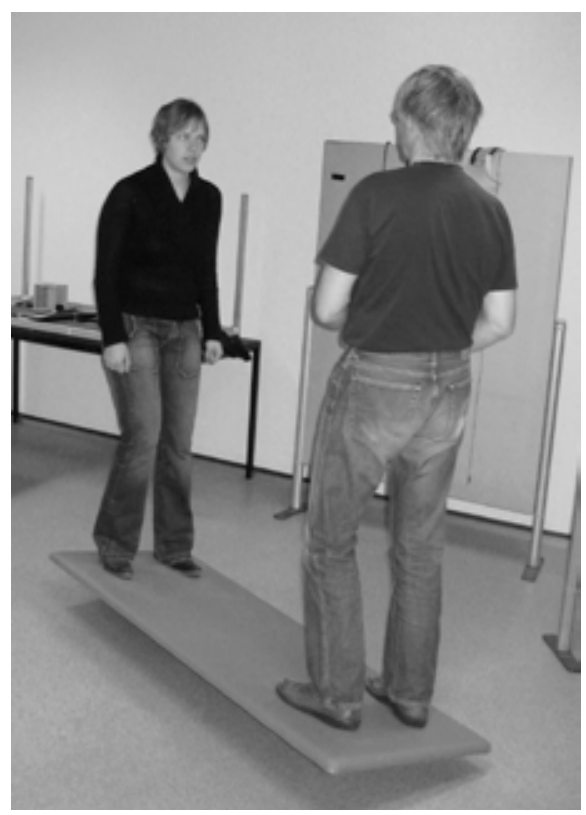

Figure 1 - Side-view of the experimental setup shows two subjects standing vis-à-vis on the rocking board, performing the task together. 
for proprioceptive training to enhance stability in stance. It compels subjects to channel the movements of their many-degrees-of-freedom movement system into a one-degree-of-freedom rocking movement (Bernstein, 1967).

A variety of studies have demonstrated how biological systems, at different levels of motor control, extract from a large set of available parameters the minimum number of parameters that are needed to act adequately. One strategy is to initially "freeze out" a portion of the available mechanical degrees-of-freedom, i.e., by allowing no or very little movement in a subset of joints (cf. Vereijken et al., 1992a). Another possibility involves optimization of certain biophysical or behavioral cost functions (reviewed in Seif-Naraghi \& Winters, 1990). Also the temporary coupling of actuators into motor synergies by moving two or more joint complexes in close phase relations has been suggested as a strategy by which the central nervous system might resolve redundancy (Cole \& Abbs, 1986; Santello, Flanders \& Soechting, 1998; D’Avella, Saltiel \& Bizzi, 2003; Ivanenko, Grasso, Zago, Molinari, Scivoletto, Castellano, Macellari \& Lacquaniti, 2003). Finally, a way in which redundancy can be managed is by relying on hierarchical control. Historically, evidence for the existence of hierarchical motor control was obtained in behavioral experiments (e.g., Rosenbaum, 1991) that yielded relevant computational principles (Arbib, Iberall, \& Lyons, 1985). Recently, in a review by Grafton and Hamilton (2007), support for action hierarchies has been provided by functional brain imaging studies. In this review, three functional brain imaging studies of action observation using the method of repetition suppression are used to identify a putative neural architecture that supports action understanding at the level of effector kinematics, object-centered movement goals and ultimately, action outcomes. These results, based on movement observation, may match a similar functional-anatomic hierarchy for action planning and execution.

In general, hierarchically managed systems rank and organize the control dimensions of a motor task at multiple levels, where each dimension of the system is a subordinate to a higher dimension and the highest dimension. The advantage of hierarchically managed systems is that they include subsystems that can be controlled independently, which simplifies the process of control tremendously. In multiarticular limb pointing and reaching movements, Dounskaia (2005) proposed the principle that there is one "leading-joint" that creates a dynamic foundation for motion of the entire limb. For example, reaching out with the hand to pick-up an object will not only result in torques that bring about the goal-directed extension of the upper and lower arm but also in reactive torques that are the consequence of intersegmental dynamics. In this context it is relevant to note that the inertia of the hand is considerably smaller than that of the upper arm and forearm. Consequently, torques at the wrist will have less effect on the motion of more proximal joints than the other way around (Soechting, 1984). According to Dounskaia's (2005) Leading Joint Hypothesis ( $\mathrm{LJH})$ the movement of the "leading-joint" generates substantial interaction torques at the other (subordinate) joints. The role of the subordinate control structures is to regulate these interaction torques such that the resulting, net torque will result in the motion of the end-effector required by the task. Consequently, while the "leading-joint" is responsible for the production of the global characteristics of the limb movement it is largely independent of subordinate joint motion thereby reducing complexity and movement variability. The subordinate joint control is relatively more complicated because it should take into 
account limb motion produced by the "leading- joint", which requires continuous coordination of this control with interaction torques. Not completely taking into account interaction torques inevitably results in higher movement variability. In sum, the "leading-joint" will (1) display features of joint rotations that contribute mainly to end-effector displacements, (2) leads in the chain of joint rotations, and (3) show low movement variability.

In the current study we use these three characteristics of the "leading-joint" to gain more insight into the principles governing intra- and interpersonal coordination in a collaborative motor task. More specifically, we tested the generality of the LJH in a kinematic analysis of the joint-coordination patterns that dyads displayed while moving a rocking board along a prescribed angle and at an imposed frequency. For within-subject (intrapersonal) interjoint coordination we expected that actors would, after having settled-in into the task, quickly resort to a "leading-joint" coordination strategy. Observations from the Vereijken et al., 1992a study showed that, on average, knee rotations $(\sim 47.90 \mathrm{deg})$ were larger than rotations at the hips $(\sim 28.66 \mathrm{deg})$ and ankles ( $23.67 \mathrm{deg})$ when participants performed slalom-like ski movements on a ski apparatus. Although coupling strength between congruent joint-rotations (hips, knees and ankles) in this study were comparable, we expected that an alternation of left and right knee rotations would be the main contributors to displace the lateral movements of the rocking board.

Rather than calculating the propelling and interaction torques separately to identify the prime movers, we reasoned that (1) the amplitudes of the joint rotations, (2) the maximum cross correlations with associated time-lag of these rotations, and (3) the variability of the continuous relative-phase analysis between congruent body rotations, would reveal which coordination strategy the subjects used. With respect to the within-subject coordination strategy, we expected to be able to identify "leading-joint" features of within-subject, congruent (e.g., left knee-right knee) joint rotations. Furthermore, in keeping with the LJH predictions we expected for the interpersonal coordination strategy that these "leading-joint" features would be allocated more often to one of the dyad partners thereby revealing a "LeaderFollower" strategy adopted by the mechanically linked collaborating dyads.

\section{Method}

\section{Participants}

Twenty-eight psychology students from the University of Nijmegen participated in our study. Their age ranged between 22 and 27 years. All participants had normal or corrected-to-normal vision and none had motor problems. All participants gave their informed consent and were rewarded for their participation with either course credits or payment of 12 Euros. Experimental procedures followed the APA guidelines for the ethical treatment of human participants.

\section{Task and Procedure}

The participants were randomly paired and given written instructions before the experimental session began. The subject-pairs (dyads) stood on a $200 \times 60 \mathrm{~cm}$ wooden rocking board with the base pad covered in nonslip surface as is custom- 
ary used in physiotherapy for proprioceptive training (see Figure 1). The board could only rock from side to side in one dimension ( $\mathrm{x}$-dimension) with a maximum tilt of 30 degrees to either side. Each participant performed side-to-side rocking movements in nine conditions spanning three amplitudes (8, 18 and 28 degs) and three frequencies $(0.4,0.6$ and $0.8 \mathrm{~Hz})$. Furthermore, the task was executed both individually and as a dyad. When together, they were placed in one of two different stances viz.: vis-à-vis (facing each other) or back-to-back (not facing each other). In both conditions, the instructed and realized amplitude-frequency combinations were presented real-time on computer displays (see Figure 2) in the form of rotating bars. Participants were asked to track the indicated movement amplitude and frequency by jointly rocking the board sideways while continuously receiving visual feedback of the actual amplitude and frequency. They were not allowed to talk to each other. Before the experiment started, participants were allowed to practice the task a few times to get comfortable with controlling the movements of the rocking board.

Each experimental session consisted of four blocks of 27 trials leading to a total of 108 trials for each session. Each trial lasted 30s. The trial started when the stimulus bar began to tilt and ended when the stimulus bar ceased to rock. Each block consisted of three repetitions of the nine amplitude-frequency combinations that were presented at random. The first and fourth trial block was always a joint-action condition either with participants facing each other or standing back-to-back. In the second and third block, the participants performed the task individually (control conditions). All blocks were counterbalanced across the experiment. Participants never performed more than 54 trials in succession.

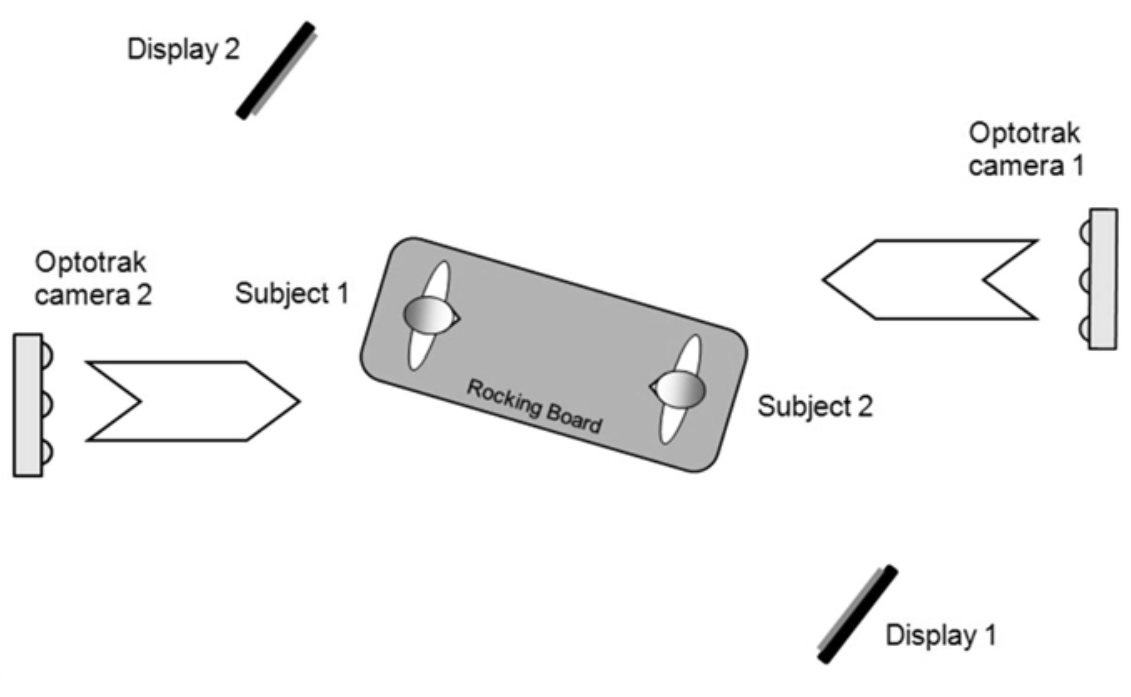

Figure 2 - Schematic top view of experimental setup showing a dyad standing on the rocking board. Two OPTOTRAK cameras were fixed at opposite sides on the walls while facing downward at an angle of $45^{\circ}$ relative to the ceiling. Two computer displays were placed at eye-level at a comfortable distance off-center to the right of the subject-pairs. 


\section{Data Acquisition}

Three rigid bodies were used: one was mounted on the base pad of the rocking board and two were strapped onto the foreheads of each participant. Each rigid body consisted of four infrared light emitting diodes (IREDs) fixated on a flat aluminum plate. In addition, 16 IREDS were attached to the ankles, knees, hips, and shoulders (over the Coracoid process) of each actor. Translations of the IREDS and rotations of the rigid bodies were recorded at a rate of $75 \mathrm{~Hz}$ and with a spatial accuracy better than $0.2 \mathrm{~mm}$ in the $x, y$ and $z$ direction by means of a 3D-motion tracking system (Optotrak 3020, Northern Digital Inc., Waterloo, Canada; Figure 2 ). At the same time, the instructed and realized angular rotations of the rocking board were sampled real-time at a rate of $38.7 \mathrm{~Hz}$ and recorded into a separate file.

\section{Data Analysis}

All position data were filtered with a second-order Butterworth, zero phase lag, low-pass filter with a cut-off frequency of $6 \mathrm{~Hz}$. The instructed and realized angular rocking movements were resampled to $75 \mathrm{~Hz}$ and filtered with a second-order, dual-pass Butterworth filter. The high-pass frequency was $0.5 \mathrm{~Hz}$ for these signals and the low-pass cut-off frequency of the filter was also set to $6 \mathrm{~Hz}$. This ensured that an automatic peak-to-peak detection algorithm could be applied reliably. On the basis of this algorithm, successive cycles were extracted of which the first and last cycle of the trial were not included in the analysis.

\section{Kinematics}

Extrinsic plane-independent, intrinsic body angular excursions (MJ $\theta$ in degs) for the left and right body side were extracted off-line from the calculated positions of the twenty-eight IREDs. The head, shoulder, hip, knee and ankle angles were defined as the enclosed angles between two neighboring segments (cf. Vereijken et al., 1992a). Extrinsic plane-independent angular displacements indicate combined rotations in three-dimensional joint space, e.g., increasing angular rotations at the hip joints indicate a combined extension/abduction/exorotation of the hip. An angle of 180 degrees at the knee joint indicates full extension.

\section{Time Series Analysis}

Continuous relative-phase time functions and maximum cross-correlation functions with associated time lag were determined for: (1) within-subject congruent intrinsic body angles (between both shoulders, hips, knees and ankles angles of a participant), (2) within-subject vertically linked intrinsic body angles (for the left and right body side of a participant) and (3) between-subject congruent intrinsic body angles (between the shoulders, hips, knees and ankles angles of each participant). The means ( $\mathrm{M} \varphi$ in degs) and standard deviations ( $S D \varphi$ in degs) of the continuous relative-phase signals of the relevant joint rotations were calculated by using Batschelet's (1981) procedure involving circular statistics (see Meulenbroek, Thomassen, van Lieshout \& Swinnen, 1998). The time lag (ms) was determined 
by shifting one of the angular displacement functions in time to obtain a maximum cross-correlation value.

The following procedure was applied to our data to provide us with an opportunity to investigate the Leading Joint Hypothesis. First, the time lag of within-subject vertically linked intrinsic body angles was determined per trial. Joint rotations that were leading in time for the duration of each trial were identified and tagged "leading-rotations" while the joints that lagged behind were tagged "followingrotations". Next, this information was transferred to both the within-subject and between-subject congruent intrinsic body angles data sets. Thus, we could now compare the variability of the phase relationships between the "leading-rotations" joints and the "following-rotations" joints. Finally, we tagged subjects as "leader" when aforementioned "leading-joint" features could be allocated more often to them than to their partner ("follower").

The critical value for statistical significance was set at the .05 level. Paired samples $t$ tests were used to evaluate the statistical significance of observed differences between the incidences of time lags after having tested for normality using the One-Sample Kolmogorov-Smirnov Test to justify using the paired $t$ test. Repeated-measures ANOVAs were applied to evaluate the continuous movement parameters.

\section{Results}

\section{Realized Amplitudes and Frequencies}

An ANOVA with imposed amplitude (8, 18 and 28 degs), imposed frequency (0.4, 0.6 and $0.8 \mathrm{~Hz}$ ), vision (solo, vis-à-vis and back-to-back) and repetition (1st, 2nd and $3 \mathrm{rd}$ ) showed that participants produced, on average, the instructed amplitudes $(\mathrm{F}(2,26)=1174.63, p<.001)$ and frequencies well $(\mathrm{F}(2,26)=2289.79, p<.001$; Table 1). The main effect of imposed amplitude on realized frequencies was significant $(\mathrm{F}(2,26)=4.49, p<.05)$. Post hoc analyses showed that participants performed the smallest amplitude ( $8 \mathrm{degs}$ ) at a lower frequency than for the larger amplitudes (18 and 28 degs). Also the main effect of imposed frequency on realized amplitudes was significant $(\mathrm{F}(2,26)=4.46, p<.05)$. Post hoc analyses showed nonlinear effects of imposed frequency on realized amplitudes. Realized amplitudes were lower for the lowest $(0.4 \mathrm{~Hz})$ and highest $(0.8 \mathrm{~Hz})$ frequency than for the $0.6 \mathrm{~Hz}$ imposed frequency. In contrast, the main effect of vision and repetition on realized amplitudes $(\mathrm{F}(2,26)=.05$, ns.; $\mathrm{F}(2,26)=2.26$, ns. $)$ and frequencies $(\mathrm{F}(2,26)=.13$, $\mathrm{ns} ; \mathrm{F}(2,26)=.98$, ns.) were not significant.

The vision (vis-à-vis and back-to-back) and repetition (1st, 2nd and 3rd) conditions were pooled in the remaining data analyses.

\section{Joint Amplitudes}

Table 2 shows the mean plane-independent angular displacements (MJ $\theta$ in degs) for all body and rocking board rotations as a function of the three imposed amplitudes (8, 18 and 28 degs).

On average, the angular displacements at the head $(\mathrm{M}=2.52, S D=1.15)$ and shoulder $(\mathrm{M}=2.49, S D=1.32)$ were relatively small across the three imposed amplitudes while the rotations at the ankle $(\mathrm{M}=4.00, S D=3.17)$ and hip $(\mathrm{M}=6.51$, 
Table 1 Left-hand panel: instructed and realized mean amplitudes (standard deviations between brackets) pooled across frequency conditions. Right-hand panel: instructed and realized mean frequencies (standard deviations between brackets) pooled across amplitude conditions.

\begin{tabular}{lllll}
\hline Amplitude (deg) & & & Frequency $\mathbf{( H z )}$ & \\
\cline { 1 - 2 } \cline { 5 - 5 } Instructed & Realized & & Instructed & Realized \\
\hline 8 & $8.89[2.21]^{*}$ & & 0.4 & $0.48[0.06]^{*}$ \\
18 & $15.85[2.37]^{*}$ & & 0.6 & $0.66[0.06]^{*}$ \\
28 & $21.14[3.04]^{*}$ & & 0.8 & $0.85[0.06]^{*}$ \\
\hline
\end{tabular}

$* \mathrm{p}<.05$

Table 2 Mean plane-independent angular displacements (MJ $\theta$ in degs; standard deviations between brackets) for all body and rocking board rotations as a function of the three imposed amplitudes (8, 18 and 28 degs).

\begin{tabular}{lccc}
\hline & $\mathbf{8 ~ d e g}$ & $\mathbf{1 8} \mathbf{~ d e g}$ & $\mathbf{2 8 ~ d e g}$ \\
\hline Head & $2.24[1.10]$ & $2.55[1.14]$ & $2.78[1.16]$ \\
Shoulder & $2.12[1.29]$ & $2.52[1.27]$ & $2.83[1.30]$ \\
Hip & $4.48[2.30]$ & $6.77[3.17]$ & $8.38[3.47]$ \\
Knee & $10.76[5.14]$ & $19.41[6.20]$ & $24.36[7.33]$ \\
Ankle & $2.32[1.97]$ & $4.30[2.99]$ & $5.71[3.52]$ \\
Rocking Board & $8.88[2.21]$ & $15.85[2.37]$ & $21.14[3.04]$ \\
\hline
\end{tabular}

$S D=3.41$ ) were relatively larger than rotations at the head and shoulder, increasing in size with increasing amplitude constraints. Even though the knee's rotation axis was orthogonally oriented to that of the rocking board, the knee produced, on average, the largest rotations $(\mathrm{M}=18.14, S D=8.43)$ that scaled proportionally with the amplitude constraints. These angular displacements at the knee were on average slightly, but significantly, larger than the rotations at the rocking board (M $=15.26, S D=5.63 ; \mathrm{t}(27)=3.678, p<.05)$.

Not only did rotations at the knee closely match the angular displacements of the rocking board in size, knee rotations were, as presented in the following results, also mostly ahead in time for all movements.

\section{Coordination Dynamics}

Figure 3 displays the incidence of number of occurrence of the means (per trial) of the continuous relative-phase time functions $(\mathrm{M} \varphi)$ for the within-subject congruent body angles. Two distinct coordination modes, in phase ( 0 degs) and out of phase (180 degs), can be observed between congruent body angles. The top-left 

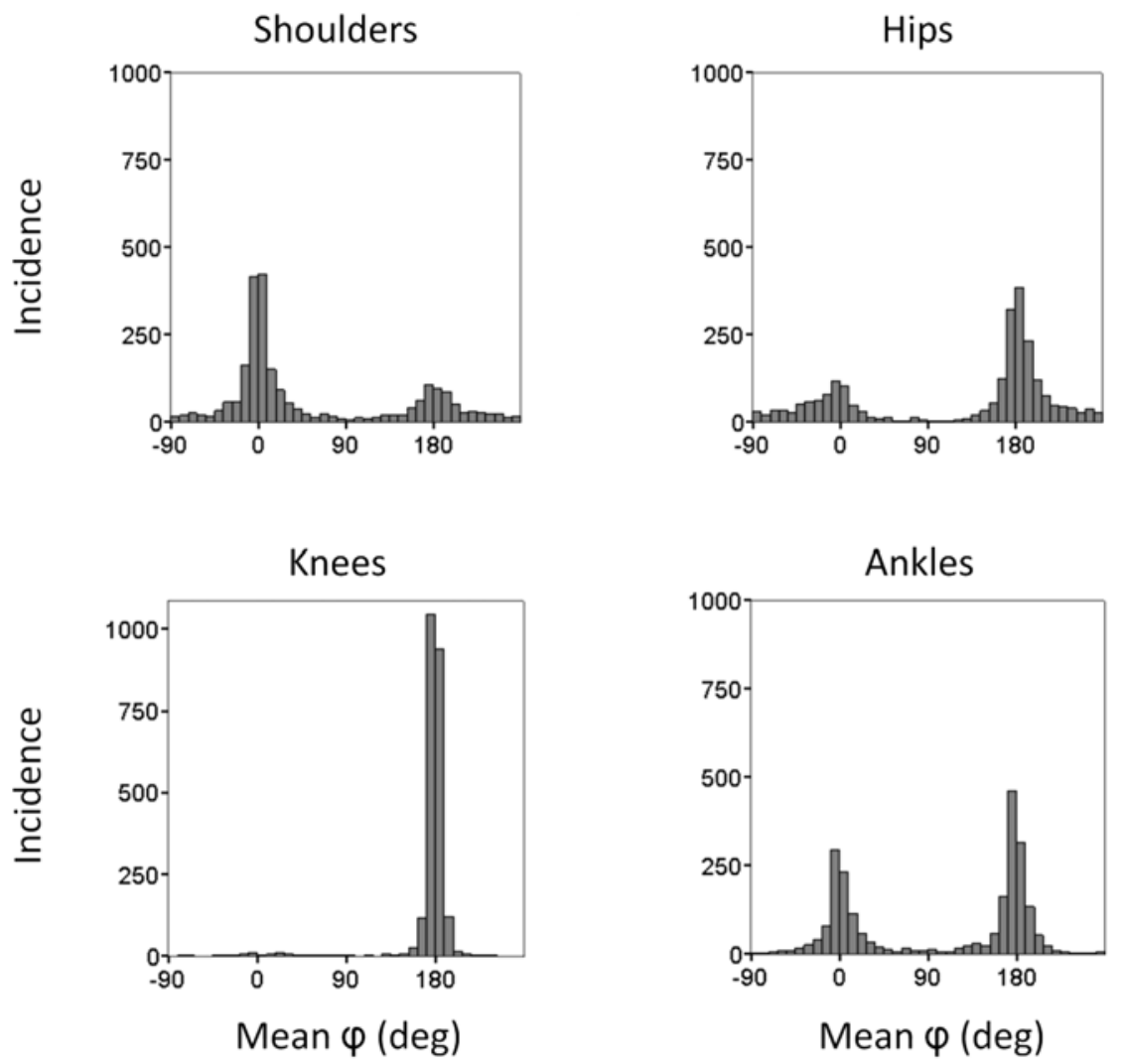

Figure 3 - Histogram displaying the incidence of the $\mathrm{M} \varphi$ (in degs) for the within-subject congruent body angles. 0 degs indicates in phase and 180 degs indicates out of phase coordination modes.

panel shows that the isodirectional (same direction) coordination mode (in phase) occurred more often than the nonisodirectional (opposite direction) coordination mode (antiphase) between the shoulder rotations. The top-right panel shows that the opposite was true for the hip rotations, i.e., antiphase was the predominant coordination mode. The bottom-left panel displays the coordination modes for the knee rotations. This plot shows that the predominant coordination mode between knee rotations during task performance was nonisodirectional, i.e., alternately flexing and extending the knees. The bottom-right panel shows no prevailing coordination mode between ankle rotations.

\section{“Leading-Rotations"}

The data of the time-lagged cross-correlations for the within-subject vertically linked intrinsic body angles was normally distributed. Figure 4 displays the number of times (in percent) a rotation at a particular location (Head, Shoulder, Hip, Knee 


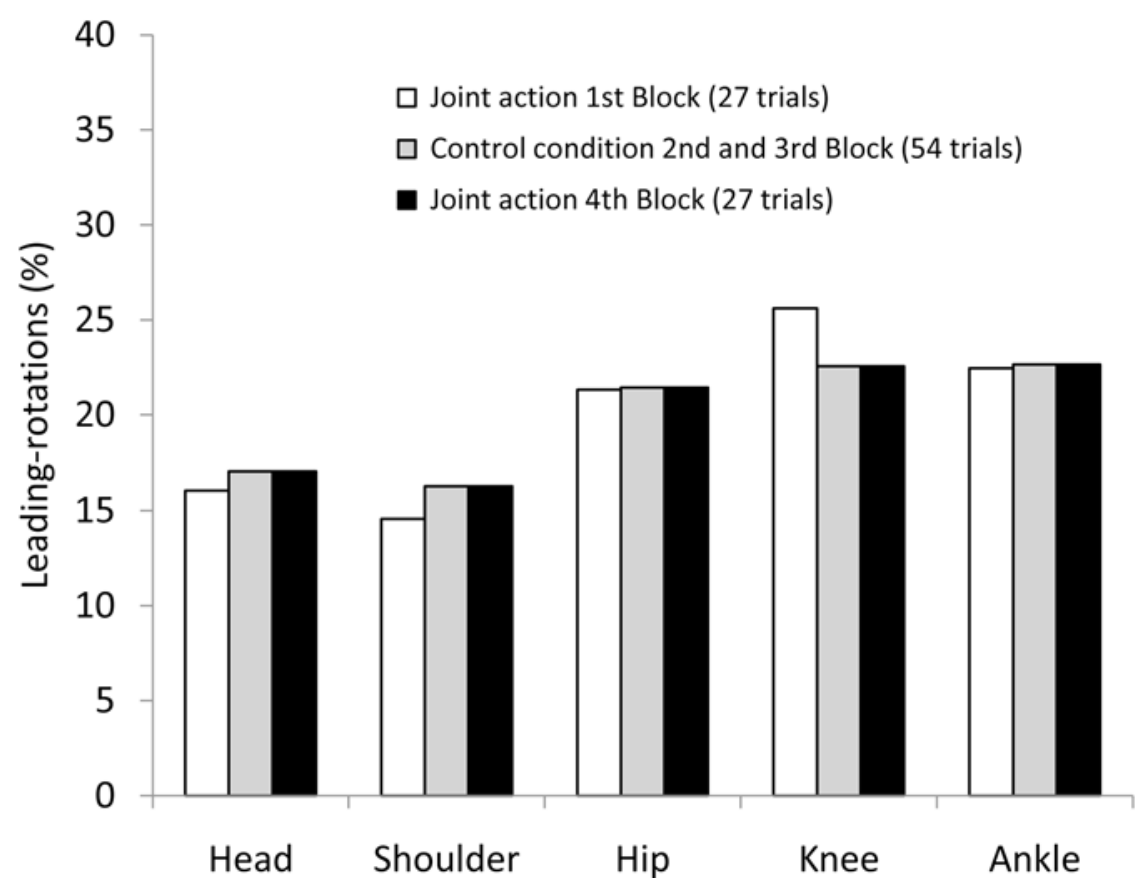

Figure 4 - Bar chart displaying the number of cases (in percent) a body rotation at a particular location (Head, Shoulder, Hip, Knee and Ankle) was ahead in time with respect to the other rotations within the blocked trials (first, second and third, and fourth block).

and Ankle) led the way in time within the blocked trials (first, second and third, and fourth block) for aforementioned dataset.

The white bars in Figure 4 show, that the incidence for the head (16\%) and shoulder $(15 \%)$ rotations leading the movement were statistically indistinguishable $(\mathrm{t}(27)=1.503, \mathrm{~ns})$. The incidence of hip rotations $(21 \%)$ ahead in time was significantly higher than the head $(\mathrm{t}(27)=6.498, p<.01)$ and shoulder $(\mathrm{t}(27)=7.460$, $p<.01)$ rotations. In addition, the ankle rotations $(22 \%)$ led the way more often than the head $(\mathrm{t}(27)=6.713, p<.01)$ and shoulder $(\mathrm{t}(27)=8.103, p<.01)$ rotations but the incidence between the hip and ankle rotations leading in movement were statistically indistinguishable $(\mathrm{t}(27)=0.999, \mathrm{~ns})$.

Knee rotations led the way in about $26 \%$ in all trials with respect to the remaining rotations viz., head $(\mathrm{t}(27)=6.419, p<.01)$, shoulder $(\mathrm{t}(27)=9.351, p<.01)$, hip $(\mathrm{t}(27)=3.459, p<.05)$ and ankle $(\mathrm{t}(27)=2.506, p<.05)$ rotations. In summary, knee rotations led other rotations most of the time while rotations at the hip and ankle led the way over the head and shoulder rotations.

The gray bars in Figure 4 show the results for the solo action conditions (block $2 \& 3$ ) in which the incidence for the head (17\%) and shoulder (16\%) rotations leading in movement were statistically indistinguishable $(\mathrm{t}(27)=.758$, ns). The incidence of hip (21\%), knee $(23 \%)$ and ankle $(23 \%)$ rotations ahead in time were significantly higher than the head (hip/head; $\mathrm{t}(27)=3.890, p<.05$; $\mathrm{knee} / \mathrm{head} ; \mathrm{t}(27)=3.660, p<.05$; ankle/head; $\mathrm{t}(27)=4.809, p<.01)$ and shoulder 
(hip/shoulder; $\mathrm{t}(27)=4.791, p<.01 ;$ knee/shoulder; $\mathrm{t}(27)=4.750, p<.01$; ankle/ shoulder; $\mathrm{t}(27)=6.293, p<.01)$ rotations. The incidence of the hip, knee and ankle leading the way were mutual statistically indistinguishable (hip/knee; $\mathrm{t}(27)=.770$, $\mathrm{ns}$; hip/ankle; $\mathrm{t}(27)=.961$, ns; knee/ankle; $\mathrm{t}(27)=.075$, ns). Taken together, rotations at the head and shoulders lagged behind in time to the leading group of rotations (hip, knee and ankle rotations).

The results for the joint condition in block 4 are displayed as black bars in Figure 4 . These bars show that the incidence for the head (17\%) and shoulder $(15 \%)$ rotations leading in movement were statistically indistinguishable $(\mathrm{t}(27)=1.522$, ns). The incidence of hip (22\%), knee (23\%) and ankle (23\%) rotations ahead in time were significantly higher than the head (hip/head; $\mathrm{t}(27)=8.030, p<.01 ; \mathrm{knee} /$ head; $\mathrm{t}(27)=4.842, p<.01$; ankle/head; $\mathrm{t}(27)=5.010, p<.01)$ and shoulder (hip/ shoulder; $\mathrm{t}(27)=8.173, p<.01 ; \mathrm{knee} /$ shoulder; $\mathrm{t}(27)=7.174, p<.01$; ankle/shoulder; $\mathrm{t}(27)=8.616, p<.01)$ rotations. Leading angular displacements at the hip, knee and ankle were statistically indistinguishable (hip/knee; $\mathrm{t}(27)=.893$, ns; hip/ankle; $\mathrm{t}(27)=.137$, ns; knee/ankle; $\mathrm{t}(27)=.905$, ns). In summary, rotations at the hip, knee and ankle mostly led the way with respect to the head and shoulder rotations.

\section{Movement Variability}

Table 3 displays the standard deviations (SD $\varphi$ in degs) of the continuous relativephase signals of the within-subject and between-subject congruent body angles during joint action.

Within-subject rotations between the knees displayed relative more stable phase relationships than rotations between the shoulders $(\mathrm{t}(13)=10.235, p<.01)$, hips $(\mathrm{t}(13)=9.498, p<.01)$ and ankles $(\mathrm{t}(13)=6.449, p<.01)$. The $S D \varphi$ between the hips were lower than the $S D \varphi$ between the shoulders $(\mathrm{t}(13)=2.751, p<.05)$ and ankles $(\mathrm{t}(13)=2.184, p<.05)$ while the variability of the phase relationships between the ankles and between the shoulders were statistically indistinguishable $(\mathrm{t}(13)=.192, \mathrm{~ns})$.

Table 3 Mean SD of the continuous relative-phase signals (SD $\varphi$ in degs; standard deviations between brackets) of the within-subject (left column) and between-subject (right column) congruent body angles as a function of body rotation (Heads, Shoulders, Hips, Knees and Ankles).

\begin{tabular}{lcc}
\hline & Within-Subject & Between-Subject \\
& $S D \varphi$ & $S D \varphi$ \\
\hline Heads & - & $73.87[4.63]$ \\
Shoulders & $63.75[11.17]$ & $73.28[5.82]$ \\
Hips & $58.48[12.77]$ & $56.86[14.64]$ \\
Knees & $42.02[13.95]$ & $34.93[17.10]$ \\
Ankles & $63.29[13.46]$ & $59.61[13.70]$ \\
\hline
\end{tabular}


Variability of the phase relationships for the between-subject congruent rotations between the knees were lower than the variability between the heads $(\mathrm{t}(13)=13.681, p<.01)$, shoulders $(\mathrm{t}(13)=13.954, p<.01)$, hips $(\mathrm{t}(13)=8.418, p<$ $.01)$ and ankles $(\mathrm{t}(13)=7.426, p<.01)$. Noteworthy, the $S D \varphi$ of the knee rotations was significantly lower between subjects than within subjects $(\mathrm{t}(13)=4.782, p<$ $.01)$. Phase relationships between the hips displayed more stable phase relationships than the $S D \varphi$ between the heads $(\mathrm{t}(13)=9.658, p<.01)$ and shoulders $(\mathrm{t}(13)=8.296$, $p<.01)$ while the $S D \varphi$ between the hips and between the ankles were statistically indistinguishable $(\mathrm{t}(13)=1.017, \mathrm{~ns})$. Furthermore, the $S D \varphi$ between the ankles was lower than the $S D \varphi$ between the heads $(\mathrm{t}(13)=7.689, p<.01)$ and shoulder $(t(13)=7.336, p<.01)$ while variability of the phase relationships between the heads and between the shoulders were statistically indistinguishable (t $(13)=1.017, \mathrm{~ns})$.

In summary, the stability of the phase relationships during joint action were highest between the knee rotations for both within-subject and between-subject congruent body angles. In addition, between-subject knee rotations were coordinated relatively more stable than within-subject knee rotations.

Figure 5 displays the standard deviations of the continuous relative-phase signals (SD $\varphi$ in degs) of the within-subject congruent body angles as a function of "leading-rotations" and "following-rotations" (see Method Section). "Leadingrotations" between the knees displayed relative more stable phase relationships than "following-rotations" between the knees $(\mathrm{t}(13)=2.833, p<.05)$. Also the $S D \varphi$ of the

\section{Joint action}

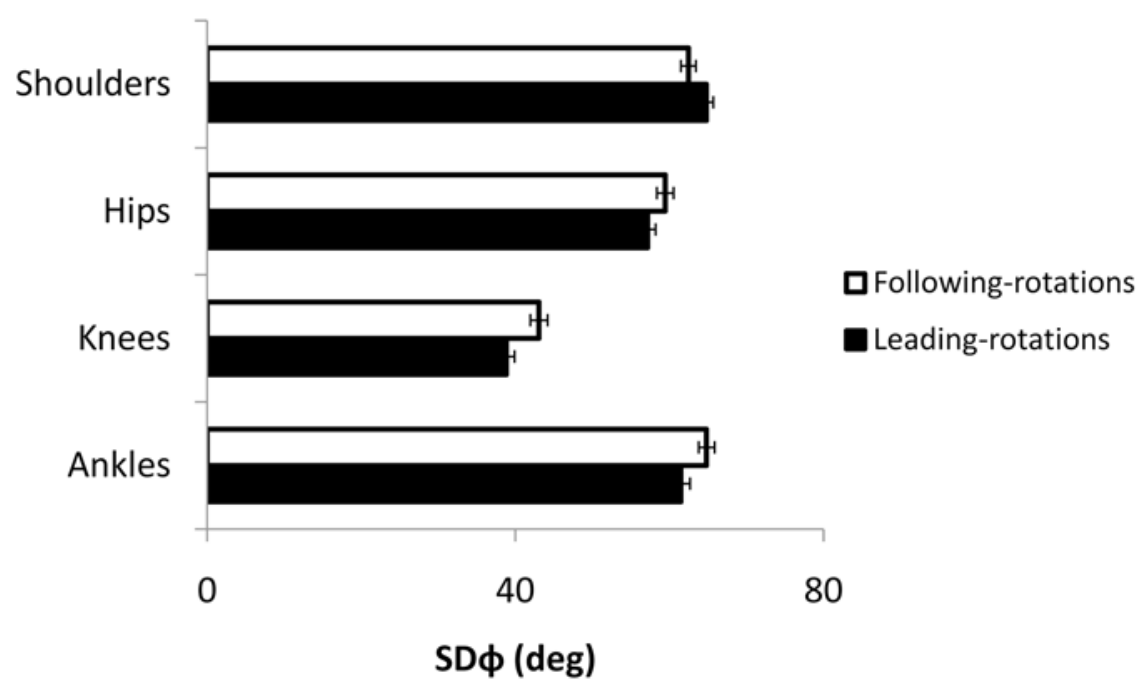

Figure 5 - Bar chart displaying the standard deviations ( $S D \varphi$ in degs) of the continuous relative-phase signals of the within-subject congruent body angles as a function of 'leadingrotations' and 'following-rotations' is shown. Error bars represent 95\% confidence intervals. 
"leading-rotations" between the ankles were lower than the $S D \varphi$ of the "followingrotations" between the ankles $(\mathrm{t}(13)=2.731, p<.05)$. In contrast, variability of phase relationships between "leading-rotations" and "following-rotations" of the hip $(\mathrm{t}(13)=1.260, \mathrm{~ns})$ and shoulder $(\mathrm{t}(13)=1.336, \mathrm{~ns})$ were statistically indistinguishable.

\section{“Leader-Follower”}

In this last section, we will report the results that allocate prime mover features to individuals. Figure 6 shows the percentage of knee rotations, which led the way while displaying low variability of phase relationships between the knees, and assigned to one of the dyads' partners. The top panel shows that, during the first block of 27 joint action trials, one of the partners displayed more features of a "leader" $(\sim 87 \%)$ than qualities of a "follower" $(\sim 13 \%$; $\mathrm{t}(13)=7.242, p<.01)$. In the last block of 27 joint action trials, "leader" features could be allocated more often $(\sim 68 \%)$ of the cases to of one partners as opposed to "follower" features $(\sim 32 \%$; $\mathrm{t}(13)=3.679, p<.05)$.

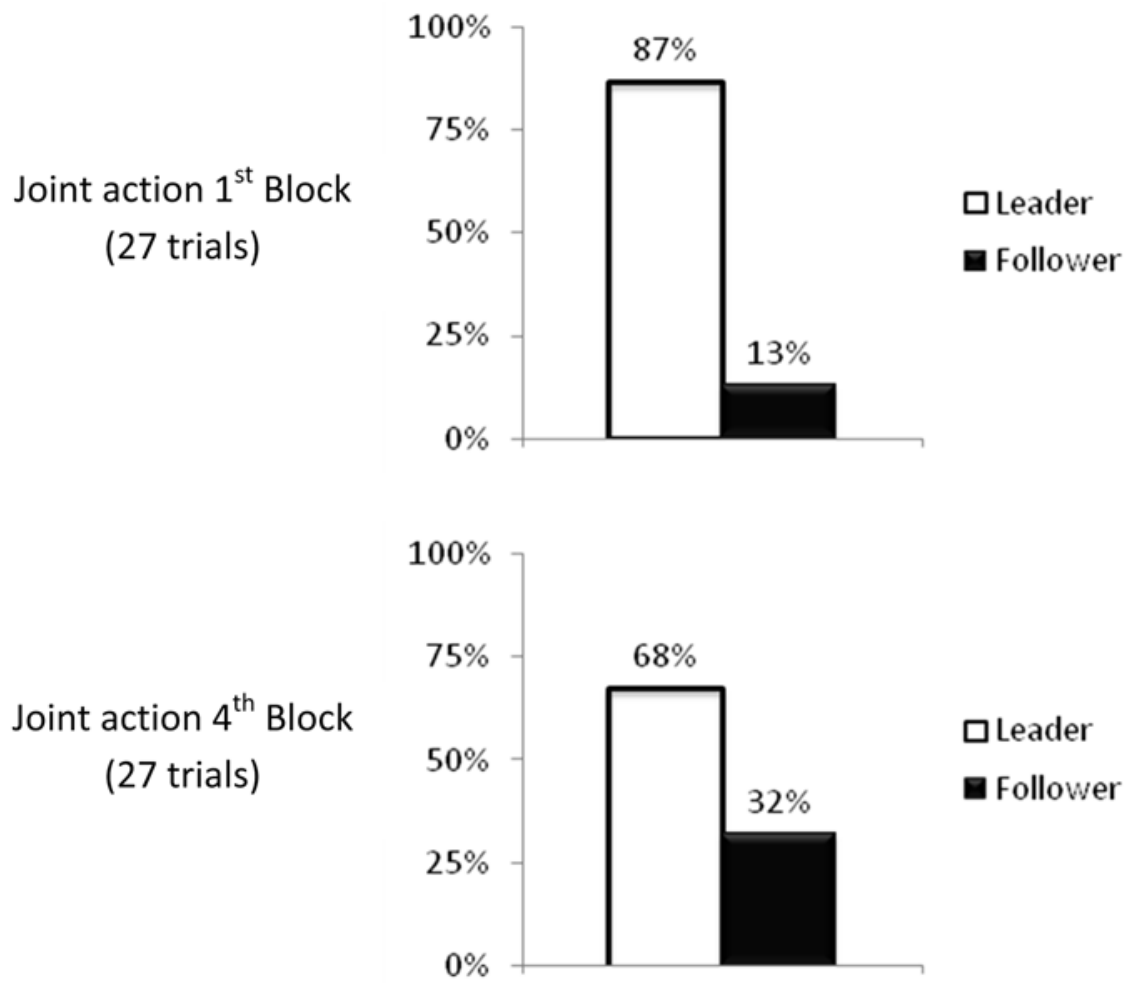

Figure 6 - Bar chart showing the number of cases (in percentage) that knee rotations displaying 'leading-joint' features could be assigned to one of the dyads' partners. 


\section{Discussion}

In this study, we tested the generality of the LJH formulated by Dounskaja (2005) as a potential motor control principle governing multijoint effector displacements. To this end we conducted a kinematic analysis of the joint-coordination patterns that two mechanically linked dyads displayed while moving a rocking board along prescribed amplitude and imposed frequency combinations. To execute the sideto-side rocking task on the rocking board, participants were forced to control their many mechanical degrees-of-freedom such that a one degree-of-freedom rocking movement resulted.

Our observations show that one way in which dyads managed redundancy was by relying on hierarchical control at both the intrapersonal and interpersonal coordination level. At the intrapersonal level, we found that knee rotations initially led all other joints while the antiphase coordination between the knees displayed relative low variability. A novel finding is that these "leading-joint" features of the knees provided dyads with a dynamic foundation to perform with respect to the coordination demands of the joint-action task. The present findings support the generality of the $\mathrm{LJH}$, in which knee rotations create a dynamic foundation at both intra- and interpersonal levels involving subordination of individual action to joint performance.

According to the $\mathrm{LJH}$, leading joints will at least display the following features viz., be the main contributor to the end-effector displacements, lead the chain of joints in time, and display the lowest movement variability.

\section{Realized Amplitudes and Frequencies}

While rhythmically moving the rocking board sideways, energy optimization most likely prompted the dyads to stick to a general strategy of amplitude undershooting. This is in line with the findings by cf. Gordon et al. (1994). The presently selected movement frequency range elicited overall frequency overshoots, probably because the preferred frequency of the rocking board, with two adults balancing on top of it, was higher than we inferred during the piloting phase of this study. The latter might also be the reason why the realized frequencies increased with an increase of the imposed amplitudes (see Bosga, Meulenbroek \& Cuijpers, 2007).

One of the questions we addressed in Bosga et al. (2007) was whether we could differentiate between incidental and deliberate control of rocking-board movements by the dyads. The study capitalized on the paradigm that we applied in a loop writing study in which we demonstrated that cycle-to-cycle amplitude/frequency parameter changes can be categorized as either intentional or biomechanical (Bosga, Meulenbroek \& Rosenbaum, 2005). Given the key role which the visual modality is supposed to play in interpersonal movement coordination (Schmidt \& O'Brien, 1997), we contrasted two conditions (haptic/visual feedback, haptic feedback) that we expected would modulate the extent to which dyads could exert deliberate control over their task performance. The results demonstrated that exploitation of biomechanics in goal-directed task performance is a prominent motor control mechanism that seems to be independent of the modalities used for monitoring the perceptual consequences of the generated motion patterns. 


\section{Joint Amplitudes}

Relative-phase analysis of intrapersonal, between-congruent body angles revealed that the predominant coordination mode between knee rotations during task performance consisted of alternately flexing and extending the knees (Figure 3). Of course predominantly, because flexion and extension of the knee joint are not simple hinge movements that occur about a fixed transverse axis of rotation but rather about a constantly changing center of rotation, that is, polycentric rotation. However, knee rotations around the main transverse axis have a normal range of motion (ROM) of about $140 \mathrm{degs}$, and were found to be, on average, the largest rotations (18 degs) in this task, scaling proportionally with the amplitude constraints, which were slightly larger than the average angular displacements of the rocking board (15 degs; see also Table 2). In-phase and out-of-phase were the dominant coordination modes for the intrinsic rotations between the shoulders, hips and ankles. This means that the rotations between these congruent joints were performed simultaneously either in the opposite directions or in the same direction, thereby allowing for a more versatile control structure than for the rotations between the knees. The hip joint is classified as a triaxial joint having movement capabilities around three orthogonally oriented rotation axes. Even though the ROM about the horizontal axis of the hip ( $155 \mathrm{degs})$ is comparable to the ROM of the knee joint around the same axis, hip rotations in this task were nearly three times smaller than knee rotations averaging $\sim 7 \mathrm{degs}$ across the amplitude constraints. The ankle presumably formed the interface between the flexion/extension movements at the knee and sideways rocking movements of the board. Rotations at the ankle joint were on average $\sim 4$ degs. Angular displacements at the head ( 3 degs) and shoulder (3 degs) were, on average, within a small range, stable and increased slightly with increasing amplitude demands.

Kinematic studies in three dimensions of natural or simulated locomotion have shown that head angular displacements in the sagittal plane remain within a range of less than five degrees (Pozzo, Berthoz, \& Lefort, 1989; 1990; Berthoz \& Pozzo, 1988; Pozzo, Berthoz, Lefort, \& Vitte; 1991). Head and trunk rotations were also shown to be relatively small and stable in tasks requiring the maintenance of equilibrium on a beam or on a rocking platform (Pozzo, Levik, \& Bertoz, 1995). On the rocking platform, the subjects tried to keep the mean angular position of the trunk near the vertical, while the lower limbs behaved like actuators of the head-trunk unit.

Not only did rotations at the knee closely match the angular displacements of the rocking board but, as we will discuss in the next section, knee rotations also initially led all movements.

\section{“Leading-Rotations”}

Time lags between intrapersonal vertically linked intrinsic body angles (Figure 4) show that there was a systematic timing order between categories of rotations. In effect, the hip, knee and ankle rotation complex led the rocking movements in about $69 \%$ of the time while the head and shoulder complex followed in time.

A popular theory of serial order holds that plans for behavioral sequences are structured hierarchically. According to this theory, the highest-level representation of 
a sequence to be produced corresponds to the sequence's main constituents, lowerlevel representations correspond to lower-level constituents, and so on (MacKay, 1982). An appealing feature of the hierarchical theory is that it accords with the fact that skill learning progresses from simple to more complex routines and that it provides a convenient way of combining and altering movement constituents at different stages (for a review, see Rosenbaum, 1987).

Our observations show that that the behavioral sequences of rotations in the task are embedded in a flexible organization structure that changes in the course of the experiment. Knee rotations in joint performance were, over the first trial block, initially ahead of all other rotations in $26 \%$ of the cases (Figure 4, white bars). Rotations of the hip (21\%) and ankle (22\%) joints led the movement less often than the knee.

Over the last trial block (Figure 4, black bars), rotations at the hip, knee and ankle rotation complex still lead the rotations of head and shoulder complex in $68 \%$ cases but rotations at the hip (22\%), knee $(23 \%)$ and ankle $(23 \%)$ equally played their part in leading the movements. These joint action results over the last trial block are comparable with the data of individual performance (see Figure 4, gray bars). Keep in mind that none of the participants had ever seen a rocking board, let alone stand on it, so they were performing a novel task. Participants evidently explored and initially controlled the movement dynamics of the task in the first 27 trials by imposing a preponderant leading-knee strategy. This strategy eventually evolved into a shared modus of hip, knee and ankle rotations leading the way in time. The predominant leading-knee strategy at the outset of the experiment undeniably simplifies the process of control by reducing redundancy but at the same time diminishes flexibility of movement production thereby placing a disproportionate burden on involved structures. We could speculate that energy optimization most likely prompted dyads to change their initial strategy over time and distribute the load over movement structures by adopting a less stringent hierarchically managed system thereby gaining movement flexibility into the bargain. The obvious advantage of being metabolically effective, by some functional measure of effectiveness leads to the prediction that people or animals would likely be reasonably effective at the things they do over time. However, we must take into account that people may have complex control strategies that generate different preferences in different circumstances. Even if energy, say, is optimized it could be that the different constraints alter the postures adopted and thus the dynamics and the energetics of the task at hand (Bertram \& Ruina, 2001).

Now we have identified the hip, knee and ankle rotations as contenders for the position of a leading joint, we will now discuss our findings concerning movement stability of these three possible candidates in which we arrived at the identity of the prime mover.

\section{Movement Variability}

"Leading-joint" control is simple and largely independent of subordinate joint motions. Subordinate joint control, however, is more complicated because it includes limb motion due to the movements of the leading joint, which requires continuous coordination at this control level. Consequently, movement variability will be lower at the "leading-joint" than at the subordinate joints. 
Analysis of the standard deviations ( $S D \varphi$ in degs) of the continuous relativephase signals during joint action show (see Table 3 ) that the $S D \varphi$ for within-subject congruent body angles between knees amounted to 42 degrees while the $\operatorname{SD} \varphi$ between the hips (58 degs) and ankles (63 degs) were markedly higher. In addition, the $S D \varphi$ for between-subject congruent body angles between the knees (35 degs) are also lower than the $S D \varphi$ between the hips (57 degs) and ankles (60 degs).

Noteworthy, the $S D \varphi$ of the congruent body angles for the knees was significantly lower for interpersonal (35 degs) than for intrapersonal (42 degs) coordination, implying that between-subject knee rotations were coordinated relatively more stable than within-subject knee rotations. This hints at the fact that during joint action participants were attuned in coordinating their actions between each other thereby implying subordination of individual action to joint performance. Even though researchers have shown that mechanisms underlying action coordination are internalized (Allport, 1993; Bekkering, Wohlschlaeger, \& Gattis, 2000; Hommel, Müsseler, Aschersleben, \& Prinz, 2001), it is interesting to note that groups of individuals can work toward a common goal without internal mechanisms (Steiner, 1972). Under these conditions, dyads might time their actions in response to each other's behavior or plan and execute their actions in relation to what they anticipate the other team members will do. However, effective coordination requires dyads to learn and use an anticipatory coordination strategy (ACS; Knoblich \& Jordan, 2003; Bosga \& Meulenbroek, 2007). In the current study we therefore presume that the dyads were capable of executing tightly coupled knee rotations at the interpersonal level because they could rely on relative fast haptic feedback to use an ACS to develop a stable base for their cooperation. From the LJH perspective, coupling of the knee rotations at the intrapersonal level is comparable with more the complex subordinate joint control and coupling is therefore relatively weaker than coupling of knee rotations at the interpersonal level.

We can confidently infer that interactions between knee rotations act as stable boosters of the rocking movements. However, the above results merely reflect features of knee rotations in a general way, i.e., averaged behavior over all trials. In the next section, we will pinpoint features to knee rotations that actually display their prime joint characteristics per trial.

As we have reported in the Method Section, we identified joint rotations that led ("leading-rotations") and those that lagged other rotations in time ("followingrotations") and subsequently determined the variability for those phase relationships in congruent joint rotations per trial.

As expected, the standard deviations of the continuous relative-phase signals (SD $\varphi$ in degs) of the within-subject congruent body angles (Figure 5) showed that knee rotations were coordinated more stable when they were "leading-rotations" (40 degs) than when they were "following-rotations" (44 degs). These results show that knee rotations displayed the largest excursions, led the way in time, and were coordinated mutually relatively very stable providing evidence that knee rotations can be regarded as the prime movers in the rocking board task.

\section{“Leader-Follower"}

Our results show that during the first block of 27 joint action trials, "leading-joint" features could be tagged in $87 \%$ of the cases to one of the dyads partners (see Figure 6). In the last block of 27 joint action trials, these "leading-joint" qualities could 
still be allocated in about $68 \%$ of the cases to of one of the partners. This means that dyads maintained a "Leader-Follower" strategy throughout the course of the experiment i.e., the dyads developed a cooperative strategy such that one member contributed more in boosting the rocking board sideways than the other. This does however not imply a linear cause-effect sequence between the "Leader" and the stable coordination at the intrapersonal knee level. For individual movements it is generally known that movement goals form a powerful regulating factor allowing people to automatically compensate for any external perturbation (Conditt, Gandolfo, \& Mussa-Ivaldi, 1997; Hasan, 2005; Bizzi, Accornero, Chapple, \& Hogan, 1984). Analogously, behavioral goals of joint action create a common ground for both actors that guide the selection of their actions (Todorov \& Jordan, 2002) and allow them to jointly reach their goals effectively. We speculate that the actors created a shared "internal model" of the dynamics of the joint action of which they are part of thereby allowing the intrinsic dynamics of joint action itself to cause and maintain the stable coordination at the intrapersonal level.

The present findings confirm our expectations that control mechanisms for dyads rhythmically moving the rocking board sideways can be modeled in line with principles of the Leading Joint Hypothesis. Dyads displayed leading-joint features at the intrapersonal level that were subordinate to the "Leader-Follower" strategy at the interpersonal level. This study extends theoretical insights in the literature on motor control to joint action. This is achieved by demonstrating that motor control principles that have been postulated for individual motor control also apply to joint action.

\section{Acknowledgments}

The present study was supported by the EU-Project "Joint Action Science and Technology" (IST-FP6-003747).

\section{References}

Allport, A. (1993). Attention and control: Have we been asking the wrong questions? In D.E. Meyer \& S. Kornblum (Eds.), Attention and performance XIV: Synergies in experimental psychology, artificial intelligence, and cognitive neuroscience (pp. 183-218). Cambridge, MA: MIT Press.

Arbib, M.A., Iberall, T., \& Lyons, D. (1985). Coordinated control program for movements of the hand. In Goodwin, A.W., \& Darian-Smith, I. (Eds). Hand function and the neocortex. Experimental Brain Research Supplement, 10, 111-129.

Batschelet, E. (1981). Circular statistics in biology. London: Academic Press.

Bekkering, H., Wohlschlaeger, A., \& Gattis, M. (2000). Imitation of gestures in children is goal-directed. Quarterly Journal of Experimental Psychology: Human Experimental Psychology, 53(A), 153-164.

Bernstein, N. (1967). The coordination and regulation of movements. London: Pergamon. Berthoz, A., \& Pozzo, T. (1988). Intermittent head stabilization during postural and locomotory tasks in humans. In Amblard, B., Berthoz, A., \& Clarac, F. (Eds). Posture and gait: Development adaptation and modulation (pp 189-198). Elsevier, Amsterdam N.Y.I Oxford.

Bizzi, E., Accornero, N., Chapple, W., \& Hogan, N. (1984). Posture control and trajectory formation during arm movement. The Journal of Neuroscience, 4, 2738-2744.

Bertram, J.E.A., \& Ruina, A. (2001). Multiple walking speed-frequency relations are predicted by constrained optimization. Journal of Theoretical Biology, 209, 445-453. 
Bosga, J., \& Meulenbroek, R.G.J. (2007). Joint-action coordination of redundant force contributions in a virtual lifting task. Motor Control, 11, 235-258.

Bosga, J., Meulenbroek, R.G.J., \& Cuijpers, R.H. (2007). Interpersonal Movement Coordination in Jointly Moving a Rocking Board. In N. Gantchev N (Ed.) From Basic Motor Control to Functional Recovery. Sofia Bulgary, pp. 36-43.

Bosga, J., Meulenbroek, R.G.J., \& Rosenbaum, D.A. (2005). Deliberate control of continuous motor performance. Journal of Motor Behavior, 37(6), 437-446.

Carlton, L.G. (1981). Processing visual feedback information for movement control. Journal of Experimental Psychology. Human Perception and Performance, 7, 1019-1030.

Cole, K.J., \& Abbs, J.H. (1986). Coordination of three-joint digit movements for rapid finger-thumb grasp. Journal of Neurophysiology, 55, 1407-1423.

Conditt, M.A., Gandolfo, F., \& Mussa-Ivaldi, F.A. (1997). The motor system does not learn the dynamics of the arm by rote memorization of past experience. Journal of Neurophysiology, 78, 554-560.

D’Avella, A., Saltiel, P., \& Bizzi, E. (2003). Combinations of muscle synergies in the construction of a natural motor behavior. Nature Neuroscience, 6, 300-308.

Dounskaia, N. (2005). The internal model and the leading joint hypothesis: implications for control of multi-joint movements. Experimental Brain Research, 166, 1-16.

Gordon, J., Ghilardi, M.F., Cooper, S.E., \& Ghez, C. (1994). Accuracy of planar reaching movements. Experimental Brain Research, 99, 112-130. [AUQ6]

Grafton, T.S., \& Hamilton, A.F. de C. (2007). Evidence for a distributed hierarchy of action representation in the brain. Human Movement Science, 26, 590-616.

Hasan, Z. (2005). The Human Motor Control System's Response to Mechanical Perturbation: Should It, Can It, and Does It Ensure Stability? Journal of Motor Behavior, 37, 484-493.

Hommel, B., Müsseler, J., Aschersleben, G., \& Prinz, W. (2001). The theory of event coding (TEC). The Behavioral and Brain Sciences, 24, 849-937.

Ivanenko, Y.P., Grasso, R., Zago, M., Molinari, M., Scivoletto, G., Castellano, V., et al. (2003). Temporal components of the motor patterns expressed by the human spinal cord reflect foot kinematics. Journal of Neurophysiology, 90, 3555-3565.

Johansson, R.S., \& Birznieks, I. (2004). First spikes in ensembles of human tactile afferents code complex spatial fingertip events. Nature Neuroscience, 7, 170-177.

Knoblich, G., \& Jordan, J.S. (2003). Action coordination in groups and individuals: Learning anticipatory control. Journal of Experimental Psychology. Learning, Memory, and Cognition, 29, 1006-1016.

MacKay, D.G. (1982). The problem of flexibility, fluency, and speed-accuracy trade-off in skilled behavior. Psychological Review, 89, 483-506.

Paillard, J. (1996). Fast and slow feedback loops for the visual correction of spatial errors in a pointing task: a reappraisal. Canadian Journal of Physiology and Pharmacology, 74, 401-417.

Pozzo, T., Berthoz, A., \& Lefort, L. (1989). Head kinematics during various motor tasks in humans. In J. Allum \& M. Hulliger (Eds.), Progress in brain research (Vol. 30, pp. 377-383). Amsterdam: Elsevier.

Pozzo, T., Berthoz, A., \& Lefort, L. (1990). Head stabilization during various locomotor tasks in humans. I. Normal subjects. Experimental Brain Research, 82, 97-106.

Pozzo, T., Berthoz, A., Lefort, L., \& Vitte, E. (1991). Head stabilization during various locomotory tasks in humans. II. Patients with peripheral vestibular deficits. Experimental Brain Research, 85, 208-217.

Pozzo, T., Levik, Y., \& Bertoz, A. (1995). Head and trunk movements in the frontal plane during complex dynamic equilibrium tasks in humans. Experimental Brain Research, 106, 327-338.

Reed, K., Peshkin, M., Hartmann, M.J., Grabowecky, M., Patton, J., \& Vishton, P.M. (2006). Haptically Linked Dyads. Are Two Motor-Control Systems Better Than One? Psychological Science, 17, 365-366. 
Rosenbaum, D.A. (1987). Successive approximations to a model of human motor programming. In G.H. Bower (Ed.), Psychology of learning and motivation (Vol. 21, pp. 153-182). Orlando, FL: Academic Press.

Rosenbaum, D.A. (1991). Human motor control. San Diego: Academic Press.

Santello, M., Flanders, M., \& Soechting, J.F. (1998). Postural hand synergies for tool use. The Journal of Neuroscience, 18, 10105-10115.

Saunders, J.A., \& Knill, D.C. (2003). Humans use continuous visual feedback from the hand to control fast reaching movements. Experimental Brain Research, 152, 341-352.

Schmidt, R.C., \& O'Brien, B. (1997). Evaluating the dynamics of unintended interpersonal coordination. Ecological Psychology, 9, 189-206.

Seif-Naraghi, N.E., \& Winters, J.M. (1990). Optimized strategies for scaling goal-directed dynamic limb movements. In: Winters, J.M \& Woo, S.L.-Y. (Eds.) Multiple muscle systems: Biomechanics and movement organization (pp. 312-334). New York: SpringerVerlag.

Soechting, J.F. (1984). Effect of target size on spatial and temporal characteristics of a pointing movement in man. Experimental Brain Research, 54, 121-132.

Steiner, I.D. (1972). Group process and productivity. New York: Academic Press.

Todorov, E., \& Jordan, M. (2002). Optimal feedback control as a theory of motor coordination. Nature Neuroscience, 5, 1226-1235.

Vereijken, B., Van Emmerik, R.E.A., Whiting, H.T.A., \& Newell, K.M. (1992a). Free(z) ing degrees of freedom in skill acquisition. Journal of Motor Behavior, 24, 133-142.

Wegner, N., \& Zeaman, D. (1956). Team and individual performances on a motor learning task. The Journal of General Psychology, 55, 127-142. 